\title{
From the Editors
}

Mehrere Kontroversen haben in der letzten Zeit den akademisch-publizistischen Bereich erschüttert, und einige davon teilten die Eigenschaft, dass sie die Entscheidungen und die Verantwortung von Herausgeber*innen in den Fokus rückten. Während es ausgiebige Untersuchungen zu ethischen Fragen gibt, die nur peripher mit tatsächlichen Problemen zu tun haben, gibt es relativ wenige Veröffentlichungen zur publizistischen Ethik. In einer der wenigen empirischen Studien zur Thematik heißt es, dass Herausgeber*innen von Zeitschriften schlicht ,nicht besonders interessiert' an ethischen Fragen seien. ${ }^{1}$ Dieser Mangel an Interesse ist bedauerlich. Schließlich ist die Veröffentlichung von Zeitschriftenartikeln der wichtigste Kanal zur Verbreitung und Diskussion von Forschungsergebnissen; und noch dazu können die Arbeitsweise und die Arbeitsabläufe einer Zeitschrift und die Handlungen und Entscheidungen ihrer Herausgeber*innen entscheidenden Einfluss gerade auf die Karrieren von Nachwuchswissenschaftler*innen haben.

Wir können darüber spekulieren, warum Ethiker*innen nicht besonders motiviert sind, ihren kritischen Blick nach innen zu wenden, auf die jeweils geschätzten Informationskanäle und -plattformen. Eine plausible Erklärung dafür ist, dass die publizistische Ethik keinen hohen Status innerhalb der Disziplin genießt: In gemütlicher akademischer Runde kann die Philosophin, die sich auf die Ethik des Tötens, Sexualethik oder Verteilungsgerechtigkeit spezialisiert hat, auf Aufmerksamkeit hoffen, aber der Professor, der Bücher über publizistische Ethik schreibt, ist hingegen relativ uninteressant. Manche Bereiche der Ethik sind schlicht nicht besonders attraktiv und bieten wenig akademische Aufmerksamkeit und akademischen Status.

Ein anderer, vielleicht etwas zynischer Grund ist, dass diejenigen Individuen und Organisationen, die am ehesten in der Position wären, sich des Themas anzunehmen, den geringsten Anreiz haben, die auch zu tun. Wenn ein Professor X seinen akademischen Grad und seinen beruflichen Status durch Veröffentlichungen in hoch angesehenen Zeitschriften erlangt hat, dann würde es vermutlich $X^{\prime}$ eigener Position schaden, wenn er sich mit der ethischen Dimension von Entscheidungen darüber, was publiziert wird, beschäftigte - da $X^{\prime}$ eigene Position ja ein Resultat dieses Systems ist. Entsprechend haben auch Herausgeber*innen keinen besonderen Anreiz, Strukturen anzugreifen, zu denen sie selber gehören.

The neueren Entwicklungen und ,Skandale' im Bereich des akademischen Publizierens scheinen die Wichtigkeit einer gut durchdachten publizistischen Ethik noch

1 E. Wager, S. Fiack, C. Graf, A. Robinson and I. Rowlands, 'Science journal editors' views on publication ethics: results of an international survey', Journal of Medical Ethics 35 (2009), S. 348-353. 
zu unterstreichen. Ich möchte drei Beispiele für Kontroversen anführen, die publizistische Ethik in den Fokus rücken, obwohl dies nicht immer erkannt wurde.

Vor einigen Jahren veröffentlichte die Zeitschrift Synthese eine thematische Nummer zu Intelligent Design, ,Die Evolution und ihre Rivalen'. ${ }^{2}$ In einer Erklärung in der gedruckten Ausgabe, die zwei Jahre nach dem Erscheinen der Online-Version der thematischen Nummer veröffentlicht wurde, entschuldigten sich die Herausgeber geradezu für den Inhalt des Heftes und merkten an (meiner Meinung nach zu Recht), dass den üblichen Standards von Respekt und Höflichkeit nicht in allen Artikeln Genüge getan wurde. Obwohl die Erklärung von Artikeln in der Mehrzahl sprach, wurde allgemein der Schluss gezogen, dass sie sich nur auf einen Beitrag bezog. Aufgrund der Erklärung wurde den Herausgebern von Synthese vorgeworfen, dass sie vor der Intelligent-Design-Lobby eingeknickt seien, und ein Boykott war schnell organisiert.

Man kann darüber streiten, ob ein Boykott gerechtfertigt war, aber die eigentliche ethische Frage, die sich in dieser Kontroverse stellte, drehte sich um die Pflichten der verantwortlichen Herausgeber gegenüber den Autor*innen. War die Erklärung eine angemessene Entschuldigung dafür, dass die verantwortlichen Herausgeber die von den Gastredakteuren ausgewählten Artikel nicht noch einmal überprüft hatten; oder hatten sie es versäumt, kontroverse Artikel zu verteidigen, nachdem Druck von externen Akteuren aufgekommen war?

Genau diese Frage stand auch im Zentrum einer anderen aktuellen Kontroverse um die Zeitschrift Hypatia. Rebecca Tuvel hatte einen Artikel mit dem Titel „In Defense of Transracialism" in Hypatia veröffentlicht, der mit Abstand prominentesten Zeitschrift für feministische Philosophie. ${ }^{3}$ Schnell wurde der Artikel zur Zielscheibe scharfer Kritik, und nachdem diese Kritik einige Zeit im Internet kursiert war, wurde ein offener Brief an Hypatia gerichtet, in dem die Rücknahme von Tuvels Artikel gefordert wurde. ${ }^{4}$ Unter diesem Druck wandten sich die Mitherausgeberinnen mit einer Erklärung an die Öffentlichkeit, in der sie sich für die Veröffentlichung des Artikels entschuldigten und zum Ausdruck brachten, dass dieser niemals hätte veröffentlicht werden dürfen. Die Mitherausgeberinnen gaben so externem Druck nach, der vor allem im Internet und auf sozialen Medien erzeugt worden war, und verteidigten die Autorin und ihre Arbeit mit keinem Wort. Besonders auffällig dabei war, dass die gesamte Kontroverse sich hauptsächlich auf sozialen Medien - insbesondere Facebook - abspielte, und dass es (zunächst) keine kritische Auseinandersetzung mit Tuvels Artikel im wissenschaftlichen Forum von Hypatia gab.

Mein drittes Beispiel unterscheidet sich etwas von den anderen beiden. Als das Journal of Medical Ethics einen Artikel mit dem Titel ,Abtreibung nach der Geburt: Warum sollte das Baby leben dürfen?' veröffentlichte, hatte dies eine Reihe kritischer Reaktionen zur Folge. ${ }^{5}$ Im Gegensatz zu den bereits besprochenen Fällen waren jedoch ein Großteil dieser Reaktionen an die Zeitschrift gerichtete philosophische Repliken und nicht

2 Synthese 178:2 (2011).

${ }^{3}$ Rebecca Tuvel, 'In Defense of Transracialism', Hypatia 32:2 (2017), S. 263-278

4 'Open Letter to Hypatia', online unter: https://docs.google.com/forms/d/1efp9C0MHch_6Kfgtlm0PZ76nirWtcEsqWHcvgid12mU/viewf orm?ts=59066d20\&edit_requested=true (Zugriff am 12.08.2017).

5 Alberto Giubilini and Francesca Minerva, 'After-birth abortion: why should the baby live?', Journal of Medical Ethics 39:5 (2013), S. 261-263. 
Stellungnahmen oder Aufrufe auf sozialen Medien. ${ }^{6}$ Die ethische Frage, die sich hier stellt, ist jedoch die einer ,Lockveröffentlichung'. Eine ,Lockveröffentlichung' ist ein eigentlich minderwertiger Artikel, dessen Veröffentlichung nur dazu dient, der Zeitschrift Aufmerksamkeit zu verschaffen und damit ihre Zitationsrate zu erhöhen. Ich behaupte nicht, dass dies beim Journal of Medical Ethics der Fall war; mein Punkt ist aber der, dass eine tatsächliche Lockveröffentlichung sehr ähnlich aussehen würde. ${ }^{7}$ Eine Lockveröffentlichung regt zwar die Diskussion zu einem Thema an, allerdings zu dem Preis der Veröffentlichung eines Beitrags, der nicht hätte veröffentlicht werden sollen.

Meiner Meinung nach sieht sich der Betrieb des wissenschaftlichen Publizierens mit substantiellen Herausforderungen konfrontiert, die oft ethischer Natur sind. Die hier besprochenen Beispiele sind deshalb gewählt worden, weil sie öffentlich ausgetragene Kontroversen generierten. $\mathrm{Zu}$ diesen Beispielen können wir auch Diskussionen um open access, Voreingenommenheit in der Entscheidungsfindung von Herausgeber*innen, mangelhafte Gutachten, kommerzielles Publizieren und ,Gastbeiträge' zählen. Diese Themen bedürfen der systematischen Auseinandersetzung, nicht nur, um einen gerechten und fairen Veröffentlichungswettbewerb zu garantieren, sondern auch, um sicherzustellen, dass gute Forschung tatsächlich an die Öffentlichkeit gelangt.

In der vorliegenden Ausgabe präsentieren wir zwei Artikel. Der erste, ,Unmoralische Gesetze und gesetzlose Ethik: Recht und Tugend in Kants Rechtslehre' von Jenna Zhang widmet sich dem im Titel genannten Werk Kants. Das Verhältnis von Recht und Tugend in Kants Philosophie ist ein kontroverses Thema, und Zhangs Artikel stellt einen wertvollen Beitrag dar. Gegen die vorherrschenden Interpretationslinien argumentiert sie, dass Kants Spätwerk Die Metaphysik der Sitten eine fundamentale Unterscheidung von Recht und Tugend beinhaltet. Das Recht sei keine Implikation der Tugend, so Zhang, sondern normativ von diesem unabhängig. Damit verunmöglichen es die unterschiedlichen und getrennten Wesenskerne von Rechtswesen und Moral, juristische und politische Entscheidungen auf Kants Ethik zu gründen. Obwohl Zhang betont, dass Kants Rechtsbegriff im Wesen positivistisch sei, stellt sie auch klar, dass Recht und Moral nicht völlig getrennt seien: „Moralität ist die Strömung, auf der das Recht schwimmt und die den Anstoß für moralische Wesen gibt, zu juristisch-politischen Subjekten zu werden".

Im zweiten Artikel, ,Lohn, Verdienst und erfolgreiche Organisationen', argumentiert Shaun Young, dass verdienstorientierter Lohn für Arbeitgeber ein ethisch und ökonomisch stimmiges Prinzip ist. Die Frage danach, welche Bezahlung für Arbeitnehmer angemessen ist, ist nicht leicht zu beantworten und sorgt oft für hitzige Diskussionen unter Kollegen. Young meint, dass der relevante Faktor in der Bestimmung von Lohnniveaus die bisherigen Verdienste seien. Verdienstorientierter Lohn sei ein Ausdruck von Respekt für den Arbeitnehmer, da er diesem ermögliche, sein Lohnniveau durch eigene Anstrengung zu beeinflussen. Diese Form der Autonomie fördere einen Sinn für Verantwortung und Verantwortlichkeit beim Arbeitnehmer. Der Respekt, der in verdienstorientiertem Lohn zum Ausdruck komme, sei auch ein ethisches Argument, dass für dieses Prinzip spreche - Young entwickelt jedoch auch ein nutzenorientiertes Argument: verdienstorientierter Lohn mache den Arbeitgeber attraktiver und stelle einen

\footnotetext{
${ }^{6}$ Leider erhielten die Herausgeber*innen auch viele Drohungen und Hassmails.

7 Meiner Meinung nach war der besagte Artikel aber kein besonders guter und hätte durchaus abgelehnt werden können. Viele Ethiker*innen würden mir natürlich widersprechen.
} 
De Ethica. A Journal of Philosophical, Theological and Applied Ethics Vol. 4:2 (2017)

Anreiz für Arbeitnehmer dar, ihr Bestes zu geben. Das wiederum mache den Arbeitgeber attraktiver für ambitionierte Arbeitnehmer, was dem Arbeitgeber auch ökonomischen Nutzen bringe. Nach Young haben wir damit eine doppelte Implikation: Was ihre Lohnniveaus angeht, wird eine ethische Organisation, ceteris paribus, auch erfolgreich sein; und eine an wirtschaftlichem Erfolg und Effizienz orientierte Organisation kann ihre Ziele erreichen, ohne ethische Normen zu verletzen. 